Meta

Journal des traducteurs

Translators' Journal

\title{
Le phénomène de la culture
}

\section{Jurij M. Lotman}

Volume 37, numéro 1, mars 1992

La traduction en Russie : théorie et pratique / Translation in Russia:

Theory and Practice

URI : https://id.erudit.org/iderudit/003521ar

DOI : https://doi.org/10.7202/003521ar

Aller au sommaire du numéro

Éditeur(s)

Les Presses de l'Université de Montréal

ISSN

0026-0452 (imprimé)

1492-1421 (numérique)

Découvrir la revue

Citer cet article

Lotman, J. M. (1992). Le phénomène de la culture. Meta, 37(1), 18-28.

https://doi.org/10.7202/003521ar

Ce document est protégé par la loi sur le droit d'auteur. L'utilisation des services d'Érudit (y compris la reproduction) est assujettie à sa politique d'utilisation que vous pouvez consulter en ligne.

https://apropos.erudit.org/fr/usagers/politique-dutilisation/ 


\section{LE PHÉNOMĖNE DE LA CULTURE}

JURIJ M. LOTMAN

Université de Tartu, Tartu, Estonie

Il n'existe pas de définition universellement admise et satisfaisante des notions d'«intelligence» et de «comportement intelligent». On propose de considérer comme identiques «intelligent» (= doué de raison), d'une part, et «humain» ou «logique», d'autre part. Ainsi, pour Turing étaient intelligentes des réactions que pendant une coopération durable nous ne pouvons distinguer de celles de l'homme. Second exemple: les multiples tentatives de concevoir des modèles d'intelligence artificielle par le raffinement de procédures logiques simples (par exemple la solution de problèmes ou la démonstration de théorèmes).

Sans prétendre à une définition exhaustive et exacte et nous contentant d'une formule pratiquement acceptable, nous dirons qu'est objet pensant celui qui est à même de:

1) stocker et transmettre de l'information (il dispose de mécanismes de communication et de mémoire, d'un langage et peut construire des messages corrects);

2) effectuer des opérations algorithmiques en vue de la transformation de ces messages ;

3) formuler des messages nouveaux.

Les messages obtenus à la suite des opérations définies en (2) ne sont pas nouveaux puisqu'ils ne sont que des transformations régulières de textes de départ. Dans un certain sens, tous les messages ainsi obtenus à partir d'un même texte peuvent être considérés comme un seul texte.

C'est dire que sont textes nouveaux des textes «irréguliers», dans la mesure où ils ne relèvent pas de règles. Pourtant, dans une perspective culturelle générale, ils n'en sont pas moins utiles et nécessaires. Ils peuvent servir de point de départ à la formulation de règles d'énonciation futures. Des règles émergent ainsi à partir de textes uniques (formés accidentellement ou provenant d'une autre culture, ainsi que des textes poétiques). Ce sont des textes incorrects ou incompréhensibles mais présumés sensés.

Entre les opérations intellectuelles classées sous (1) et (2) et celles classées sous (3), il y a opposition. La communication se réalise par la transmission de messages. Elle est considérée comme optimale si elle ne cause aucune perte ou altération du sens, le texte reçu étant parfaitement identique au texte émis. Tous les changements du texte du fait de son transport sont considérés comme autant de distorsions dues à l'imperfection technique du canal ou à des perturbations. Les opérations de codage et de décodage sont symétriques. Toutes les opérations ne concernent que l'expression.

Les opérations de transformations d'un message prévues en (2) sont effectuées suivant des règles algorithmiques. C'est dire qu'elles sont réversibles: inversée, la transformation ramène au texte de départ.

Un message nouveau est, lui, le fait d'un dispositif de type foncièrement différent. Seront dits messages nouveaux ceux qui ne résultent pas de transformations univoques et, partant, ne peuvent pas être dérivés automatiquement d'un texte de départ par l'application d'un répertoire de règles. En d'autres termes, un système de type: 


$$
\begin{aligned}
& \text { objet extérieur } \\
& \text { (texte réel) }
\end{aligned}-\begin{gathered}
\text { dispositif automatique } \\
\text { de photographie }
\end{gathered}-\ldots \rightarrow \begin{gathered}
\text { texte } \\
\text { (photo) }
\end{gathered}
$$

ne crée pas, à notre sens, de messages nouveaux et, en tant que tel - quel qu'en soit le degré de complexité - ne peut servir de modèle d'un acte de pensée, même si on y incorpore un mécanisme «impulsion - action». Seule une conscience créative est capable de concevoir des pensées nouvelles. Pour restituer ce processus, un modèle d'un genre foncièrement différent s'impose.

Imaginons deux langues, $L_{1}$ et $L_{2}$, aux structures différentes au point de rendre impossible une traduction exacte. L'une est une langue à unités sémantiques discrètes, à significations constantes et à organisation syntagmatique linéaire. L'autre est une langue à organisation continue, spatiale. Les plans sémantiques des deux langues sont en conséquence organisés de façon fondamentalement différente. Une traduction exacte d'un texte de $L_{1}$ par les moyens de $L_{2}$ est inconcevable. Au mieux, on obtiendra un texte qui, par rapport au contexte culturel respectif, pourrait être considéré comme équivalent. C'est éventuellement la traduction d'un texte verbal en texte iconique, par exemple dans le langage de la peinture du XIX siècle. S'il fallait ensuite procéder à une retraduction vers $L_{1}$, on n'obtiendrait naturellement pas le texte de départ. Ce serait par rapport au texte initial un message nouveau. $C f$ :

$$
L_{1} \quad L_{2}
$$

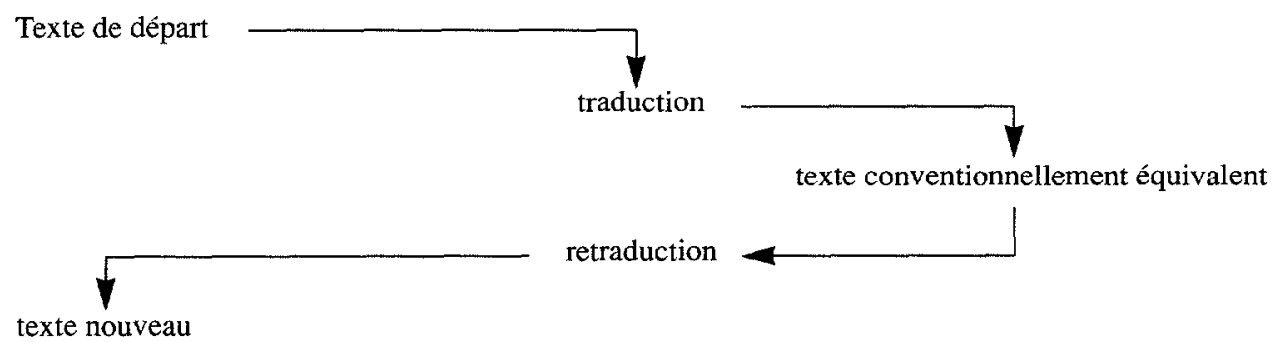

La structure de la traduction conventionnellement adéquate peut servir de modèle simplifié de créativité intellectuelle. C'est dire qu'aucun dispositif pensant ne peut être monostructuré et unilingue: il doit nécessairement inclure des formations sémiotiques multiples et irréversibles. L'hétérogénéité sémiotique est une condition sine qua non de toute structure intellectuelle.

Une structure unilingue peut rendre compte d'un réseau où circulent des messages formulés, mais pas de la conception de messages nouveaux. Pour qu'apparaisse l'incorrection légitime et naturelle qui constitue la nature d'un message nouveau ou d'une lecture nouvelle (et qui donne l'impulsion à la formation d'un langage nouveau), il faut une structure au moins bilingue. Cela explique l'énigme par ailleurs mystérieuse de l'hétérogénéité et du polyglottisme de la culture, ainsi que de tous les dispositifs intellectuels. La coexistence de langages discrets et de langages iconiques dont les signes différents ne s'alignent pas mais maintiennent des rapports d'homomorphisme qui se manifestent en symboles homologues ( $c f$. la représentation mythologique de l'homomorphisme du corps humain et des structures sociales et cosmiques) est un trait universel du dualisme structurel des cultures humaines. Bien qu'à différentes étapes de l'histoire, tel ou tel autre de ces langages universaux prétende à la globalité et puisse 
effectivement occuper une position dominante', la culture n'en demeure pas moins bipolaire, et cela sous des apparences complexes et secondaires. Bien plus, structure minimale de l'organisation sémiotique, la bipolarité se retrouve à tous les niveaux du mécanisme de la pensée, de la structure bihémisphérique du cerveau humain jusqu'à tous les niveaux de la culture.

Une seule illustration suffira. La conscience mythologique se caractérise par une vision cyclique du temps. Le cycle annuel est assimilé au journalier, la vie humaine à la végétale; naissance - mort - renaissance règnent en loi. La similitude est la loi de cet univers, l'homomorphisme, son principal rapport culturel. Automne $\simeq$ soir $\simeq$ vieillesse; conception $\simeq$ ensemencement de la terre $\simeq$ toute pénétration dans un espace sombre et clos $\simeq$ enterrement du défunt $\simeq$ engloutissement (le signe « $\cong$ marque la similitude). Par conséquent, défunt $\simeq$ «graine»; la mort est tout aussi indispensable à la résurrection que l'ensemencement à la germination; c'est par la pensée analogique que s'explique le fait que la torture, la mise en pièces du corps et l'éparpillement de ce dernier, ou encore la rupture et l'engloutissement ne soient pas autre chose que l'ensemencement et participent de ce fait à la résurrection et à la renaissance. La puissante assimilation qui sous-tend ce type de conscience fait que les diverses manifestations du monde réel apparaissent comme les signes d'une entité unique et que la diversité des objets d'une même classe se conçoit comme un seul et même objet. Toute la variété des collisions humaines est ramenée à l'histoire de la paire essentielle, l'Homme et la Femme. Unique, la Femme est à la fois Mère et Épouse de cet Homme unique. Celui-ci, à la fois Père et Fils, meurt cycliquement dans l'acte de la conception pour ressusciter dans celui de la naissance.

On retiendra que tous les textes mythologiques connus sont des transformations, des traductions de la conscience mythologique dans un langage verbal, linéaire (le mythe «réel» est iconique et spatial et manifeste sa signification dans le geste et la panchronie des dessins tels que les dessins des cavernes et les peintures rupestres, qui ne connaissent pas l'ordonnancement linéaire), des traductions aussi dans une séquence historique linéarisée. C'est là la source des notions de génération et d'étape, tous ces «d'abord» et «ensuite» qui articulent les récits écrits et oraux mais qui appartiennent proprement au mythe non pas en tant que tel mais à sa traduction en un langage non mythologique. $\mathrm{Ce}$ qui est une séquence dans un langage traduisant une pensée linéaire se présente dans l'univers mythologique en cercles concentriques en relation d'homomorphisme. Ce qui n'exclut pas qu'un personnage, unique à l'intérieur d'un cercle, puisse se décomposer en personnages antagonistes dans un autre cercle ${ }^{2}$.

Cependant, à aucun moment l'univers mythologique n'a pu être l'unique cadre de la conscience humaine (de même qu'à aucun stade les hommes n'ont pu se parler uniquement en vers pas plus qu'en ignorer jusqu'à l'existence). L'univers accidentel, celui d'événements fortuits (du point de vue du mythe) et d'actes humains sans correspondance avec les profondes lois cycliques; se constituait sous forme de récits ordonnés sur un axe temporel, linéaire. À la différence du mythe qui augurait ce qui devait se produire, l'univers accidentel faisait référence à des événements réels, opposant à la panchronie du mythe un temps passé réel et rejetant celles des lois fondamentales qui ne s'accordaient pas avec les événements observés. Sur l'axe à déroulement temporel naissent la chronique, le récit des mœurs, l'histoire.

Malgré l'antagonisme flagrant et le combat sans relâche entre ces deux modèles et langages, la vision humaine découvre l'image du monde réel à travers un système permanent de traductions et de transpositions de textes à l'intérieur du champ de tension structurel entre les deux pôles mentionnés. Tantôt elle discerne la similitude de choses d'apparence différente, des analogies et des homo- et isomorphismes pertinents pour la pensée poétique, ainsi que, partiellement, mathématique et philosophique; tantôt elle met 
en évidence des séquences, des enchaînements de causes et effets, chronologiques et logiques, qui vont nourrir les textes narratifs ainsi que les disciplines logiques et expérimentales. Ainsi, l'univers de la conscience enfantine, essentiellement mythologique, ne disparaît pas et ne doit pas disparaître dans la structure mentale de l'adulte, mais continue de fonctionner comme source d'associations et de modèles. Passer outre ce mécanisme, c'est se priver de la possibilité d'appréhender le comportement de l'adulte.

L'organisation bipolaire que l'on observe aux divers niveaux des activités intellectuelles se ramène à des oppositions binaires entre une organisation discrète linéarisée et une organisation homomorphe et continue; s'esquisse aussi un parallèle avec les principes de la pensée individuelle relevant respectivement de l'hémisphère gauche et droit:

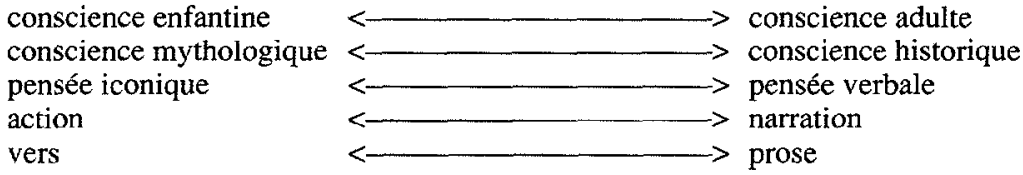

Ce système d'oppositions pourrait être prolongé. Bien plus, il importe de souligner que si dans la connaissance du monde se dégage un niveau quelconque de similitudes sémiotiques, surgit aussitôt, dans son sein, une opposition qui peut s'inscrire dans le schéma esquissé ci-dessus. Faute de quoi, le mécanisme sémiotique en question se trouve vidé de dynamisme interne, il est capable de transmettre de l'information mais est incapable d'en créer.

Que des textes d'une langue discrète ne se prêtent pas à la traduction vers une langue continue (et inversement) tient à leur structure foncièrement différente : dans la première, le texte est secondaire par rapport au signe et se décompose nettement en signes. Aussi est-il aisé d'y mettre en évidence une unité sémantique primaire. Dans les langues continues, c'est le texte qui prime. Il ne se laisse pas décomposer en signes, étant lui-même signe ou isomorphe à un signe. Ces derniers textes obéissent non pas à la syntagmatique, mais au rythme et à la symétrie (respectivement à l'arythmie et à l'asymétrie). Si l'on y dégage une unité élémentaire, elle ne se décompose pas en traits différentiels. Ainsi, s'agissant d'identifier une personne inconnue (par exemple deux photos de quelqu'un qu'on ne connaît pas), on cherchera à établir des correspondances trait par trait. Or, les textes continus (par exemple un visage familier) sont identifiés, eux, grâce à une saisie intégrale, non différenciée. L'on pourrait se référer également à la reconnaissance d'images oniriques : à travers toutes les transformations, on reconnaît sans faute la signification à attribuer à telle ou telle opposition ${ }^{3}$. Comparez les vers suivants de l'Incantation de Pouchkine (dans la version de Marina Tsvetaieva):

Apparais-moi, fantôme cher,

Comme tu fus quand nous nous dîmes

Adieu; plus pâle que l'hiver,

En proie aux affres de l'abîme.

Ou comme un souffle aérien

Ou comme un son, vivante, morte

Épouvantable - que m'importe!

- À moi, mon cœur, reviens, reviens!

Ce ne sont pas là des signes conventionnels, des liens conventionnels entre des expressions différentes (fantôme cher, son, souffle aérien) et le contenu «toi». Ces images sont autant d'hypostases dont l'apparence émane immédiatement du contenu. De même qu'en topologie, le cube est une sphère bien qu'il n'y ressemble pas, toutes ces images 
sont «toi». Dans les langages discrets, un signe se combine avec un autre signe; dans les langages continus, il se transforme en une autre manifestation de son être ou s'assimile à une trace du signe respectif à un autre niveau.

Dès lors que la différence entre les structures des langues est profonde, l'exactitude de la traduction fait place aux problèmes de l'équivalence sémantique. Toutefois, la tendance à la spécialisation poussée des langues et à la difficulté croissante de la traduction n'est qu'un aspect du processus complexe dont la somme constitue un tout intellectuel. La structure pensante doit se muer en personnalité, c'est-à-dire intégrer des structures sémiotiques opposées en un tout cohérent. En d'autres termes, les tendances opposées doivent se fondre en une structure unique pour que, malgré l'impossibilité apparente de la traduction entre $L_{1}$ et $L_{2}$ et vice versa, une telle traduction s'effectue régulièrement et donne des résultats positifs. Là où la communication entre les deux langues s'avère vraiment impossible, se produit la désagrégation de la personnalité culturelle du niveau respectif; elle cesse simplement d'exister au plan sémiotique et, parfois, aussi physique.

Deux genres de mécanismes d'intégration existent. C'est, premièrement, le bloc du métalangage. Les descriptions métalangagières sont un élément indispensable de l'ensemble intellectuel. D'une part, concevant deux langues différentes comme une seule, ces descriptions s'imposent à la vision subjective comme un tout cohérent. Orienté vers la métadescription, le système s'auto-organise, rejetant les éléments qui, dans cette optique, n'ont pas de raison d'être et faisant ressortir ce qui est retenu. Au moment de sa naissance, la métadescription est tournée vers le futur et le désirable. Avec l'évolution, elle devient réalité, norme de l'ensemble sémiotique en question.

D'autre part, les auto-métadescriptions font en sorte que l'ensemble est perçu de l'extérieur aussi comme un tout intégral auquel on attribue une certaine cohérence dans le comportement, aussi bien que dans le contexte culturel. À son tour, une telle évolution stimule l'unité de l'autoperception et du comportement de l'ensemble.

Deuxièmement, peut intervenir la créolisation des langues: les principes de l'une d'elles exercent une forte influence sur l'autre, malgré une différence fondamentale entre leurs grammaires. En pratique, le mélange des deux langues peut se produire, et cela à l'insu du sujet parlant, dans la mesure où ce dernier reconnaît sa langue à travers les métadescriptions qui, le plus souvent, naissent sur la base de l'une seulement des langues de contact. Ainsi, le russe moderne fonctionne comme un mélange de langages parlé et écrit, qui, au fond, relèvent de deux langues différentes. Ce mélange échappe au sujet parlant: la conscience métalinguistique identifie la forme écrite de la langue avec la langue tout court.

Le cinéma présente à cet égard un intérêt exceptionnel. Dès le début, il apparaît comme un phénomène bilingue (photographie mouvante + texte écrit = cinéma muet; photographie mouvante + parole = cinéma parlant ; s'y ajoute, éventuellement, mais assez souvent, un troisième langage, la musique). Or, dans la perception du public, le cinéma fonctionne comme un système unilingue. Fait significatif: bien que l'art du cinéma et celui de la dramaturgie théâtrale présentent une certaine similitude psychologique en tant que mélange de langage verbal et de langage gestuel, le public perçoit le thêâtre comme un art verbal par excellence, et le cinéma, comme une action par excellence. On comprend dès lors que la «partition» du spectacle, la pièce, fixe, essentiellement, les paroles, laissant les sons, les gestes et les actes à la compétence des acteurs (le texte verbal est invariant, celui des gestes et actes est variable), tandis que la partition d'un film, le script, fixe, en premier lieu, les actions, les événements et les gestes, c'est-à-dire le langage des images, confiant le plus souvent les paroles aux soins des dialoguistes, à moins qu'il ne laisse le champ libre à l'interprétation du réalisateur. Respectivement, les 
métadescriptions scientifiques théâtrales partent généralement des paroles, tandis que les études cinématographiques envisagent les événements visuels du langage. Le théâtre est plus proche de la littérature, fondement de son métalangage, le cinéma, de la photographie.

Cependant, nous intéresse ici la créolisation des langages constitutifs du cinéma. À l'époque du cinéma muet et des films montés, l'influence du langage oral s'est traduite dans une nette articulation du film en «paroles» et «phrases» due au transfert dans le domaine des signes iconiques du rapport conventionnel entre l'expression et le contenu. Il en découla une poétique du montage inspirée du principe de l'art verbal à l'époque du futurisme. Le langage de la photographie mouvante est devenu ainsi celui de l'art cinématographique.

À l'époque du cinéma parlant, le langage cinématographique s'émancipe énergiquement de l'emprise de l'oralité. Mais on constate en même temps un puissant mouvement en sens inverse: les techniques du film imposent des textes brefs, cependant que l'esthétique changée du film, l'abandon de la poétique du geste mimique, détournait le langage cinématographique du théâtre et de la littérature pour l'orienter vers le langage parlé. De la structure complexe du langage cinématographique, le film parlant ne retient qu'un des aspects du langage parlé, la réplique hachée, elliptique : le slang. L'intrusion de cette couche du langage dans l'art du cinéma en a relevé le prestige culturel, lui conférant une fixité culturellement équivalente à celle du langage écrit. (Le cinéma, à cet égard, est fondamentalement différent de la littérature : l'œuvre littéraire se veut une représentation du langage parlé dont elle donne une image écrite stylisée, tandis que le cinéma peut lui donner corps, lui rendre son état naturel.) Les conséquences de cette évolution ont débordé le cadre du cinéma. En effet, si précédemment bien parler, c'était parler comme dans les livres, comme au théâtre, c'est-à-dire parler de façon régulière, artificielle, «comme un livre», désormais «parler comme au cinéma», c'est, dans bien des cas, «parler comme on parle», irrégulièrement, à demi-mot. Le jargon fait désormais partie du style moderne. Visant à la spécificité, la parole s'autorise d'une norme culturelle idéale. On le voit, et d'autres exemples le confirment, les interférences langagières font que les langues réellement parlées (mais pas leurs modèles et métadescriptions) sont un mélange décomposable en systèmes sémiotiques (langages) différents.

En somme, deux mouvements opposés animent la culture. La dualité pousse à la fission ininterrompue de chaque langage culturellement acquis, multipliant sans cesse le nombre des langages de la culture. Chacun de ces langages représente un tout autonome, immanent, en même temps qu'un ensemble sémiotique, l'aboutissement d'un processus d'intégration. Chaque langage est ainsi à la fois langage et sous-langage, contexte culturel de rang supérieur et élément d'un autre contexte culturel. En cette qualité, la langue acquiert une spécification complémentaire en vertu de l'asymétrie fondamentale de la culture. Voici un exemple de ce genre d'oppositions :

$$
\begin{array}{lll}
\text { Prose littéraire } & < & \longrightarrow \text { poésie } \\
\text { Prose non littéraire } & \longrightarrow & \\
& & \text { prose littéraire }
\end{array}
$$

Il est évident que «prose littéraire» dans la première paire n'est pas identique à la même expression dans la seconde : dans le premier cas, elle manifeste le segmentaire, le discret, le linéaire propre à tout langage verbal et opposé à la poésie; dans le second, avec la poésie, et au même titre que la poésie, un élément du langage des belles-lettres. C'est uniquement à ce titre, contrairement à la prose non littéraire, qu'elle peut s'intégrer avec cette dernière dans la structure «discours en prose»: seuls peuvent s'intégrer des éléments dissemblables. La spécification sémiotique croissante est une tendance permanente dans 
l'histoire de la culture, en même temps qu'elle stimule l'intégration des langues en une culture unique.

Chaque paire sémiotique de langues intégrées, capable de recevoir, transmettre et conserver l'information et, ce qui est plus important, d'en concevoir, est une machine pensante; c'est, à certains égards, une «individualité culturelle ${ }^{4}$ ». S'intégrant à des niveaux toujours plus élevés, les «individualités culturelles» forment, au sommet, l'individualité de la culture.

La culture est proprement inconcevable en dehors de la distinction physicopsychologique entre les individus. Les théories, et elles sont nombreuses, qui opèrent avec le concept $d^{\prime}$ '«homme» le posent en modèle invariant, assez riche pour pouvoir servir à la conception de modèles socioculturels. On passe outre ce qui distingue un individu de l'autre, de même que la nature de ces distinctions. On présume que les différences entre individus sont des variables extérieures au système et sans rapport avec le modèle cognitif. Ainsi, par exemple, s'agissant du schéma élémentaire de la communication, il semble parfaitement normal de poser que l'expéditeur et le destinataire du message sont munis de codes parfaitement identiques. Et c'est ce schéma qui est pris pour modèle de l'acte communicatif réel. Certes, tout culturologue sait qu'aucun être humain n'est la réplique exacte d'un autre et en diffère par ses particularités psychophysiologiques, par son expérience, son apparence, son caractère, etc. Mais il ne s'agit là, suppose-t-on, que d'«erreurs techniques» de la nature qui, en raison de «capacités de production» limitées, n'est pas capable d'aménager une fabrication en séries, que tout ce qui relève du domaine du variable individuel est sans rapport avec la nature du phénomène social et culturel qu'est l'homme. Vision qui remonte à l'Antiquité mais qui n'a été nettement formulée que par les sociologues du Xvmr siècle. Maintes fois critiquée depuis, elle subsiste néanmoins en tant que présupposé tacite.

Nous partons de l'hypothèse opposée et supposons que les distinctions individuelles (de même que les distinctions psychologico-culturelles collectives qui se superposent) appartiennent aux fondements mêmes de l'existence humaine en tant qu'objet culturel sémiotique. C'est précisément la variation de l'individualité humaine qui est à la base des nombreux actes communicatifs et culturels.

Imaginons un organisme (une machine) qui à tous les stimulus extérieurs ne réagit que par deux réponses; admettons, par exemple, qu'il est doué de la capacité de distinguer selon le degré d'éclairage le jour de la nuit et de réagir en conséquence de deux façons différentes, par exemple de brancher une lampe en réponse au signal «nuit» et de la débrancher au signal «jour». Raccordée à un autre dispositif identique de façon à pouvoir lui transmettre les signaux «nuit» ou «jour» et lui faire brancher ou débrancher une lampe, cette machine sera douée:

1) d'omniscience. Pauvre et inefficace, notre machine ne pourra pas dispenser une information intégrale même sur le milieu environnant, bien que ce savoir soit absolu dans les limites de l'alphabet imparti. En effet, elle ne répondra toujours qu'à l'unique question prévue par son concepteur. La réponse: je ne sais pas lui est inaccessible. Dans toutes les situations, elle ne dégagera que le paramètre lumière-absence de lumière et réagira en conséquence, rejetant tous les autres paramètres comme non pertinents ;

2) d'absence de doute et d'hésitation. L'analyse du milieu extérieur et la réaction correspondante étant automatiquement couplées, il ne saurait y avoir d'hésitation dans le choix du comportement. C'est dire qu'il peut être inefficace et ne pas assurer la survie de l'organisme tout en étant extrêmement fiable. La saisie univoque de l'état du milieu entraînera une réaction univoque; 
3) d'une totale compréhension mutuelle entre l'expéditeur et le destinataire du signal. Ce système assure l'entière équivalence des textes reçus et retransmis. L'incompréhension ne peut être que le fait d'une défaillance dans le canal de transmission.

Supposons maintenant que notre dispositif doive évoluer pour développer sa capacité de survie. On serait amené dans ce cas à commencer par augmenter au maximum le nombre des paramètres de l'environnement susceptibles de déclencher une réaction. Il est toutefois évident qu'en procédant ainsi on n'obtiendra pas la mutation du dispositif : la réaction ne sera pas devenue conscience intellectuelle.

Il n'y a intelligence que si l'alphabet du code au moyen duquel le dispositif destiné à refléter le monde extérieur identifie les états de l'environnement est pourvu d'une case vide en vue d'états futurs qu'il reste à connaître et à nommer. La segmentation du monde extérieur, le décodage de ses états et la traduction de ses données dans son code de la machine ne seront plus dans ce cas fixés une fois pour toutes. Quel que soit le système de classification, il reste toujours des éléments non identifiés à connaître, à définir et à penser.

Avec l'introduction de telles «cases vides», le mécanisme qui commande la réaction de notre machine aura acquis des traits d'intelligence: souplesse, aptitude au perfectionnement, et cela grâce à un fonctionnement plus efficace et à la conception de modèles plus opératoires de saisie du monde extérieur. Elle aura perdu du mêrne coup l'omniscience, la réponse automatique à n'importe quelle question, ainsi que l'absence d'hésitation, le lien automatique entre l'information reçue et l'acte à accomplir. À cette dernière circonstance s'associe un autre fait marquant, le passage de l'automatisme à un comportement intelligent. Si auparavant à chaque stimulus extérieur correspondait une seule et même réaction, désormais deux réactions également valables à un certain égard et en un certain sens sont déclenchées, ce qui impose la formation d'un mécanisme d'appréciation et de sélection. C'est dire que d'automatique la réaction sera devenue pertinente, elle sera un acte.

L'aptitude au choix entre des réactions différentes, tout en augmentant l'efficacité de notre machine jusqu'à lui attribuer un comportement propre, comporte nécessairement l'hésitation. C'est dire que dès lors que nous avons enrichi l'organisation du dispositif au point de le douer d'intelligence, de l'aptitude à réagir de façon souple et efficace aux changements du monde environnant et de s'y orienter, à concevoir des modèles de plus en plus efficaces, il se trouve aussi placé dans un état d'ignorance et d'incertitude croissante. Ceux qui s'occupent d'intelligence artificielle ne devraient pas oublier que les dispositifs intelligents qu'ils veulent construire (à moins que l'on n'entende par là des annexes mécaniques à l'intelligence humaine) risquent de produire des êtres en proie à des névroses dues à un sentiment de frustration devant le manque d'information, au doute dans le choíx de la stratégie à adopter.

De par sa nature même, le phénomène de la pensée ne peut être autosuffisant. Comme tout perfectionnement, toute découverte, une invention, en éliminant les problèmes présents, en engendre d'autres encore plus grands. Le bond dans l'évolution de la pensée, cause de stabilité, de chances meilleures de survie dans le monde environnant, appelait de nouvelles découvertes capables de surmonter les difficultés engendrées par une existence consciente. D'une part, il n'était que trop naturel de vouloir compenser l'incertitude et l'ignorance accrues par l'invocation d'êtres protecteurs doués d'omniscience. La religion paraît avec la pensée, et ce n'est pas là un événement fortuit. Mais ce problème déborde notre propos. Disons, plutôt, que le fait d'en appeler à la raison collective, c'est-à-dire à la culture, est un autre moyen de surmonter les difficultés engendrées. La culture, l'intelligence supra-individuelle est un mécanisme qui compense les insuffisances de l'intelligence 
individuelle et lui est nécessairement complémentaire. En effet, le mécanisme de la culture peut se concevoir comme suit: l'insuffisance de l'information dont dispose l'individu pensant lui impose la recherche de la communication avec d'autres êtres : placé dans des conditions d'information totale, l'individu n'aurait guère besoin de semblables pour prendre des décisions. Or, l'homme évolue normalement dans des situations où l'information est insuffisante. Plus nos connaissances augmenteront, plus le besoin d'information ira en croissant, dépassant le progrès scientifique. Or, on ne peut parer au manque d'information que par un effet stéréoscopique: la possibilité d'obtenir une projection parfaitement différente d'une seule et même réalité par la traduction dans une langue différente. L'utilité du partenaire de la communication vient précisément de son altérité. Le profit collectif des participants à un acte de communication, c'est l'accession à des modèles qui servent à enrichir la vision du monde, enrichissement qui n'est possible qu'à condition que les codes des intelligences ne coïncident pas : la communication n'est mutuellement utile que si les participants parlent des langues différentes. Cela revient à dire qu'avec le développement de la culture se perd le troisième avantage d'un système simple: la pleine compréhension mutuelle entre les participants de la communication. Il y a plus: le mécanisme de la culture qui pousse toute personne vers une autre contribue aussi à l'essor de l'identité de chacune d'elles. La communication en devient d'autant plus difficile. Y remédient des mécanismes métalangagiers et l'éclosion d'une langue commune, mélange de sous-langages différents et spécifiques. Conservant leur identité et leur autonomie, les individualités personnelles sont intégrées dans une individualité plus complexe d'ordre supérieur: la culture.

Il est évident que le même système de rapports qui relie les langues (les structures sémiotiques différentes) en un ensemble supérieur intègre aussi des individualités différentes en un tout pensant. La conjugaison de ces deux mécanismes à structure typologique similaire forme l'intelligence supra-individuelle qu'est la culture. La marque de la culture par rapport aux ensembles supra-individuels d'ordre inférieur (de type «fourmilière»), c'est que chaque individualité qui en fait partie n'en demeure pas moins un tout. C'est pourquoi les rapports entre les éléments ne sont pas automatiques, mais supposent dans chaque pas une tension sémiotique ainsi que des collisions parfois dramatiques. Le principe de génération culturelle mentionné ici agit dans les deux sens. L'évolution de la culture conduit, d'une part, à l'éclosion, à l'intérieur de la conscience individuelle, de «personnalités» psychologiques différentes entre lesquelles se nouent de complexes liens communicatifs et, d'autre part, à l'intégration dans des ensembles sémiotiques de personnalités à potentialités élevées.

La richesse des conflits intérieurs assure à l'intelligence collective qu'est la culture une souplesse et un dynamisme extraordinaires.

Post-scriptum. Quinze ans après. L'article que nous proposons à l'attention des lecteurs de Meta date de 1975 et a paru dans «La sémiotique de la culture», Travaux sémiotiques, vol. X (Travaux de l'Université de Tartu, v. 463, Tartu, 1978: 3-17). Il est partie constitutive d'un cycle d'études unies non seulement par un lien chronologique (1970-1980) mais aussi par une problématique commune. Parmi ces problèmes, en voici deux qu'il n'est peut-être pas inutile de préciser.

1) La recherche porte sur la culture. Bien que le point de départ de l'auteur ait été l'opposition saussurienne langue-parole, la démarche au moment de la conception de la présente étude et des travaux qui s'y rattachent avait sensiblement évolué. En effet, sur le plan synchronique, débordant langue et parole, l'étude prend pour objet un erısemble sémiotique bien plus complexe. On pose tout d'abord que le fonctionnement réel de langues isolées est impossible: 
une langue isolée est une convention pédagogique et heuristique. La signification est toujours le fait d'une traduction dans une autre langue (que s'y ajoute par ailleurs une seconde composante, «la langue de la réalité», ne change rien en principe). Autrement dit, c'est la paire de langues qui est l'unité sémiotique élémentaire. Mais cet objet est, lui aussi, une pure abstraction scientifique. Système sémiotique, la culture fonctionne réellement comme un complexe de langues différemment organisées et à degré de traductibilité allant de la traductibilité absolue (traduction dans la même langue, seule concevable pour les langues artificielles) à l'intraductibilité tout aussi absolue, comme c'est le cas pour la glossolalie ou pour les formes extrêmes de l'art. Le mouvement entre la compréhension totale et l'incompréhension totale est un processus sémiotique. Un sens nouveau peut donc être défini comme le fait d'une traduction d'abord inadéquate, l'ensemble des différents systèmes sémiotiques étant une génératrice de sens.

2) La rencontre de systèmes sémiotiques se produit aussi en diachronie (historiquement). Les langues évoluant à des vitesses différentes, il est naturel qu'un examen synchronique en fasse apparaître des éléments appartenant à des structures temporelles différentes. Comme en biologie, où nous sommes confrontés à des étapes d'évolution différentes, un découpage de la culture montre des faits dont la génération remonte à des années (que l'on pense à la mode, par exemple), en même temps que des mécanismes dont le cycle se mesure en millénaires. À cela s'ajoute que les systèmes sont en corrélation avec les stades de leur évolution, avec leurs textes appartenant à des étapes passées aussi bien qu'à des états possibles dans l'avenir.

3) En somme, le système sémiotique évolue à l'intérieur de la sémiosphère (l'espace des sphères sémiotiques, réelles aussi bien qu'éventuelles, de la sémiosis). Le mouvement dans cet espace constitue un des problèmes fondamentaux de la sémiotique. Bien avant la rencontre avec les idées d'I. Prigojine, qui ont exercé sur l'école de Tartu une influence révolutionnaire, cette dernière avait avancé l'idée de l'action primordiale de la contingence historique. Nous soulignons que la description des événements passés (des processus achevés) et, d'autre part, celle de ceux en cours relèvent de principes différents. Le présent s'accomplit à travers des contingences à probabilités égales, imprévisibles en principe et de ce fait porteuses d'information. Le passé comporte des probabilités acquises et est de ce fait redondant. B. Pasternak, après Schlegel, a exprimé cette pensée en disant que l'historien est «un prophète du révolu». Le présent est le moment où l'avenir variable se transforme en causalité rigidement organisée du passé, étant entendu que le moment présent n'obéit qu'en partie aux lois de la probabilité. Dans les termes d'I. Prigojine, au moment de l'explosion domine l'imprévisible qui ne se transforme en causalité que post factum. Ajoutons que dans le domaine à structures multiples de la culture, des explosions dans l'un de ses secteurs peuvent coïncider avec une évolution à organisation rigide dans un autre secteur. étude.

C'est dans le cadre des idées que nous venons d'esquisser que se place la présente

Notes

1. Ainsi, dans la culture européenne des XVII-XIX ${ }^{\mathrm{c}}$ siècles, le système verbal et discret domine manifestement. La langue naturelle et le métalangage logique s'élèvent au rang de modèles de la culture tout court. Or, c'est précisément aux époques de domination d'un seul système qu'apparaît avec évidence l'impossibilité de son unicité. 
2. Voir Ju. Lotman (1973), «La genèse du sujet. Aspect typologique», in Articles sur la typologie de la culture, Tartu (en russe).

3. Voir la description d'un rêve par Léon Tolstoï: «Le petit vieux enferme sa tête dans la neige, et ce n'est pas tant un petit vieux qu'un lapin, et il bondit loin de nous. Tous les chiens courent après lui. Le conseiller, qui est Féodor Philippitch, ordonne que tous s'assoient en cercle [...] mais le petit vieux s'est transformé, $c$ 'est maintenant le noyé.» («Une tourmente de neige».) Les images-signes ne sont pas ici conventionnelles dans la mesure où leur expression tient inconditionnellement à leur contenu. Elles ne sont pas non plus iconiques (la transformation de l'aspect extérieur signalerait alors le passage à un autre signe: le lièvre, le noyé et le petit vieux, le conseiller et Féodor Philippitch seraient des signes différents; or, nous identifions le lièvrepetit-vieux-noyé comme un seul et même objet). L'existence même de signes conventionnels et iconiques reflète dans le système discret le dualisme «discret - non discret». Une telle transposition du dualisme sémiotique de la culture dans une de ces parties s'accompagne du doublement des signes du type verbal (signification discrète du discret), ce qui fait que pratiquement ils deviennent des méta-unités, les signes iconiques devenant, eux, des formations hybrides: signification discrète du continu.

4. Ju. Lotman (1977), La culture comme intelligence collective et les problèmes de l'intelligence artificielle, Moscou (en russe). 